\title{
Wavelength-Swept Cascaded Raman Fiber Laser around 1300 nm for OCT Imaging
}

\author{
Hyung-Seok Lee, Hwi Don Lee, Myung-Yung Jeong, and Chang-Seok Kim* \\ Department of Cogno Mechatronics Engineering, Pusan National University, Busan 609-735, Korea
}

(Received January 2, 2015 : revised March 10, 2015 : accepted March 13, 2015)

\begin{abstract}
We experimentally demonstrated a novel wavelength-swept laser using a cascaded Raman gain around $1310 \mathrm{~nm}$. A 1064/1310 wavelength division multiplexing (WDM) coupler and coupled fiber Bragg gratings mirrors at $1064,1117,1175,1240 \mathrm{~nm}$ are effectively used to increase the power efficiency in a laser ring cavity with highly non-linear fiber (HNLF) of $2 \mathrm{~km}$. Linear wavelength sweeping is demonstrated with the $100 \mathrm{~Hz}$ triangular driving signal to fiber Fabry-Perot tunable filter (FFP-TF) around the 1310 $\mathrm{nm}$ region. The measured sweeping range and output power were $27 \mathrm{~nm}$ and $2.1 \mathrm{~mW}$, respectively, which are suitable for optical coherence tomography (OCT) imaging.
\end{abstract}

Keywords: Optical coherence tomography, Fiber Raman amplifier, Wavelength-swept laser OCIS codes : (110.4500) Optical coherence tomography; (140.3550) Lasers, Raman; (060.3510) Lasers, fiber

\section{INTRODUCTION}

Wavelength-swept lasers have formed important light sources for optical coherence tomography (OCT) imaging [1-3] and fiber Bragg gratings (FBG) optical sensor applications [4]. The critical factors of swept-source OCT (SSOCT) imaging depend on the central wavelength region, spectral tuning range and sweeping speed of the wavelengthswept laser. The central wavelength region of the wavelengthswept laser is selectively decided in accordance with the biological application in question as scattering and absorption losses in tissues vary significantly with the light wavelength $[1,2]$. The spectral tuning range of the wavelength-swept laser should be increased to improve the axial resolution of SS-OCT imaging [3]. The sweeping speed of the wavelengthswept laser is also important to improve the acquisition speed of SS-OCT imaging $[5,6]$. Since the central wavelength region and spectral tuning range of the wavelength-swept laser are determined by the gain medium in the laser cavity, various gain media such as semiconductor optical amplifiers (SOAs) [1-4], erbium-doped fiber amplifiers (EDFAs) [7], ytterbium-doped fiber amplifiers (YDFAs) [8], fiber optical parametric amplifiers (FOPAs) [9], and fiber Raman amplifiers (FRAs) [10] have been employed in the last decade. The central wavelength region of an SOA can be selected from various gain windows of 800 $\mathrm{nm}, 1060 \mathrm{~nm}, 1310 \mathrm{~nm}$, and $1550 \mathrm{~nm}$. However, a broad spectral tuning range of the SOA can only be achieved with multiple SOA chips because the gain bandwidth of the SOA is limited by its material properties [4]. The EDFA and YDFA have indigenous central wavelength regions of $1550 \mathrm{~nm}$ and $1060 \mathrm{~nm}$, respectively, along with a limited spectral tuning range around their central wavelengths $[7,8]$. FOPAs can reportedly provide broad gain windows; however, this requires extraordinarily high pump power of over tens of watts [9].

Recently, we have proposed the use of the FRA to implement a wavelength-swept fiber Raman laser operating around the $1550 \mathrm{~nm}$ region using optical pumping sources in the range of $1425 \mathrm{~nm}$ to $1465 \mathrm{~nm}[10,11]$. The FRA offers advantages such as the instantaneous carrier relaxation time of stimulated Raman scattering and arbitrary gain band selection by the wavelength of the optical pump sources. However, to the best of our knowledge, most wavelength-swept fiber Raman lasers have been implemented around the 1550 $\mathrm{nm}$ region because high-power optical pumping sources are commercially available only in the range of $1425 \mathrm{~nm}$ to $1465 \mathrm{~nm}$ [10-13]. Meanwhile, $1550 \mathrm{~nm}$-centered wavelength-swept fiber Raman lasers find very little use in biomedical OCT applications because there is no specific biological merit offered by lasers operating in the $1550 \mathrm{~nm}$ region. A P-doped fiber has been used as Raman gain medium to simplify schematic design and obtain high efficiency [14-17]. In this case, however, a specific wavelength region of

\footnotetext{
*Corresponding author: ckim@pusan.ac.kr

Color versions of one or more of the figures in this paper are available online.
} 
1240 and $1480 \mathrm{~nm}$ can be obtained for the first and second Stokes respectively by the use of a $1064 \mathrm{~nm}$ pumping wavelength. Thus the P-doped fiber is not useful for the $1310 \mathrm{~nm}$-centered wavelength-swept Raman fiber laser.

In this research, for the first time, we implemented a $1310 \mathrm{~nm}$-centered wavelength-swept fiber Raman laser in the light of the preferential use of the $1310 \mathrm{~nm}$ wavelength region for dermatological OCT applications. When incident on human skin tissue, laser wavelengths centered on 1310 $\mathrm{nm}$ provide less scattering loss than those centered around $800 \mathrm{~nm}$ or $1060 \mathrm{~nm}$ and less absorption loss than that obtained with a central wavelength of $1550 \mathrm{~nm}$ [1-3]. For the implementation, an optical pumping source operating around the $1240 \mathrm{~nm}$ region is necessary for the $1310 \mathrm{~nm}$-centered wavelength-swept Raman fiber laser, but commercially available pumping power in the $1240 \mathrm{~nm}$ region is limited. Our novel cavity design yielded high FRA around the $1310 \mathrm{~nm}$ region with the use of a $1064 \mathrm{~nm}$ pumping source that provided cascaded fourth-order Raman gain. To increase the pumping efficiency, a 1064/1310 nm wavelength-division multiplexing (WDM) coupler and coupled FBG mirrors reflecting wavelengths of $1064,1117,1175$, and $1240 \mathrm{~nm}$ were optimally used in a laser ring cavity. Cascaded Raman fiber lasers can form a convenient alternative gain medium in the $1310 \mathrm{~nm}$ region as well as other wavelength regions as they provide highly efficient architecture [18].

\section{WAVELENGTH SWEPT CASCADED RAMAN FIBER LASER}

Figure 1 shows the schematic of the cascaded Raman amplifier for the $1310 \mathrm{~nm}$ gain region. The Raman amplifier consists of a $1064 \mathrm{~nm} \mathrm{Yb-doped} \mathrm{fiber} \mathrm{laser,} \mathrm{a} \mathrm{1064/1310}$ $\mathrm{nm}$ WDM coupler, a $2 \mathrm{~km}$ long highly nonlinear fiber (HNLF), coupled FBG mirrors, and an optical isolator. The original optical pumping at $1064 \mathrm{~nm}$ is generated from the continuous-wave $\mathrm{Yb}$-doped fiber laser, which has a maximum output power of $\sim 20 \mathrm{~W}$. The optical isolator is used to achieve unidirectional propagation of light in order to avoid the generation of standing waves in the resonance cavity. The coupled FBG mirror pairs that have

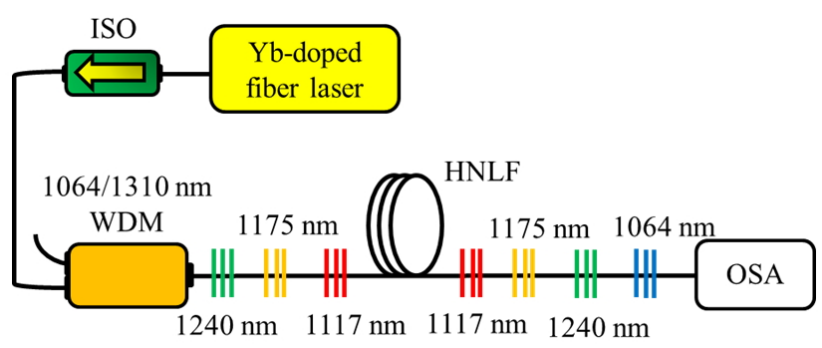

FIG. 1. Setup of the cascaded Raman amplifier based on Yb-doped laser pumping source. (WDM: Wavelength Division Multiplexing, HNLF: High Non-Linear Fiber, OSA: Optical Spectrum Analyzer, ISO: Isolator)
Bragg wavelengths of 1117, 1175, and $1240 \mathrm{~nm}$ are placed on both sides of the HNLF. The reflectivity of most FBGs was greater than $95 \%$. The $3 \mathrm{~dB}$ bandwidths of $1064 \mathrm{~nm}$, $1117 \mathrm{~nm}, 1175 \mathrm{~nm}$ and $1240 \mathrm{~nm}$ 's FBGs were 12, 0.6, 0.9 and $0.6 \mathrm{~nm}$, respectively.

In addition, an FBG that has a Bragg wavelength of $1064 \mathrm{~nm}$ is placed at the end of the laser cavity to reflect high-power optical pump light. This linear laser cavity with seven FBGs is designed to effectively generate a cascaded fourth-order Raman-Stokes gain in the $1310 \mathrm{~nm}$ region. Since a broad amplified spontaneous emission (ASE) gain around the $1310 \mathrm{~nm}$ region is necessary to realize the $1310 \mathrm{~nm}$-centered wavelength-swept fiber Raman laser, the lasing beam at $1240 \mathrm{~nm}$ (via the coupled $1240 \mathrm{~nm}$ FBG mirrors) is used as a high-power optical pumping source for ASE generation in the $1310 \mathrm{~nm}$ region. Figure 2 shows the cascaded Raman amplifier output spectra of the first-, second-, third-, and fourth-order Stokes waves. Initially, the HNLF is pumped by the Yb-doped fiber laser, and optical signals at the first Stokes frequency are generated by stimulated Raman scattering. When these signals continue to propagate along the fiber, they serve as a pump for secondorder Stokes waves. While increasing the pump power, these processes are repeated to obtain higher-order Stokes waves. From the experiment with the $2 \mathrm{~km}$ long HNLF, the cascaded first-order $(1117 \mathrm{~nm})$, second-order $(1175 \mathrm{~nm})$, third-order $(1240 \mathrm{~nm})$, and fourth-order Stokes $(1310 \mathrm{~nm})$ waves are obtained when the 1064-nm laser's pump power is 540 $\mathrm{mW}, 820 \mathrm{~mW}, 1.7 \mathrm{~W}$, and $2.3 \mathrm{~W}$, respectively.

Considering the interaction between the pump light and Stokes waves, the generation of the instantaneous Stokes intensity is proportional to the optical pumping power and the stimulated Raman gain coefficient [18-21]. With increase in the length of the HNLF, the stimulated Raman gain coefficient can proportionally increase; however, the increased fiber loss coefficient at the Stokes wavelength suppresses the generation of the instantaneous Stokes intensity. Therefore, within the limits of the given length of the HNLF and the limited optical pump power, it is important to increase the path-length efficiency by applying a feedback cavity structure.

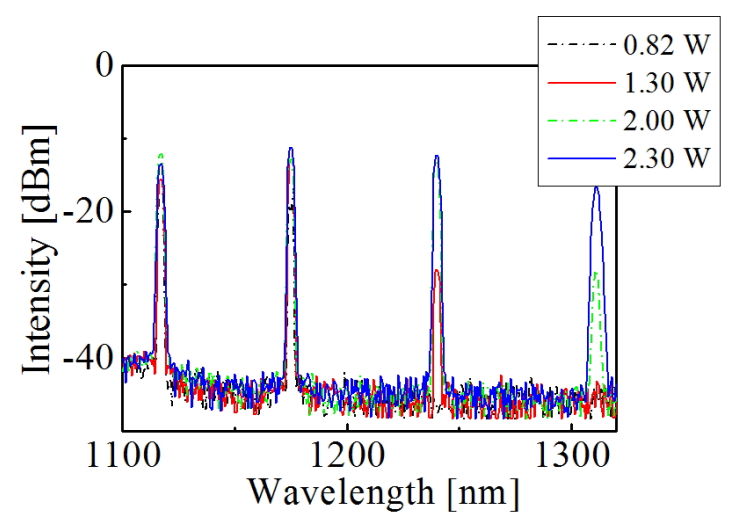

FIG. 2. The output spectra of the first-, second-, third-, and fourth-order Stokes waves. 
Since each cavity round-trip across the HNLF is optimally induced from the pairs of the FBG mirrors at 1064, 1117, 1175 , and $1240 \mathrm{~nm}$, the required pump power to increase the Stokes intensity can be effectively reduced, as can be inferred from the coupled equations of the cascaded RamanStokes shift [21]. When we remove the coupled FBG mirrors and adopt a ring cavity feedback structure based on the 1064/1310 nm WDM coupler only, a higher pumping power of more than $3 \mathrm{~W}$ is required to generate the fourthorder Stokes waves [18].

Figure 3 shows the schematic of the wavelength-swept fiber laser based on the cascaded Raman amplifier. The wavelength-swept cascaded Raman fiber laser consists of a Raman amplifier with optical gain around $1310 \mathrm{~nm}$ (Fig. 1), a fiber Fabry-Perot tunable filter (FFP-TF; Micron Optics), an 80:20 output coupler, two isolators, and a polarization controller (PC). The laser is coupled with the fiber ring resonance cavity via the 1064/1310-nm WDM coupler. Since the 1064/1310 nm WDM coupler exhibits a minimum crosscoupling ratio around 1064 and $1550 \mathrm{~nm}$ and a maximum cross-coupling ratio around $1310 \mathrm{~nm}$, it is useful to combine the optical pumping beam at $1064 \mathrm{~nm}$ into the ring cavity and to induce the highest feedback efficiency around the $1310 \mathrm{~nm}$ region in the ring cavity [18]. In this research, linear cavity feedback using the pairs of FBGs is used to reduce the required pump power to shift from $1064 \mathrm{~nm}$ to $1310 \mathrm{~nm}$, and the ring cavity feedback using the 1064/1310 $\mathrm{nm}$ WDM coupler is used to form a lasing cavity for the gain region around $1310 \mathrm{~nm}$.

The above-mentioned setup can be used to select a lasing wavelength according to a driving electrical signal. A certain wavelength around the gain region of around $1310 \mathrm{~nm}$ can be swiftly selected for lasing via the transmission window provided by the FFP-TF. Figure 4 (a) shows the output spectrum of the laser at $1310 \mathrm{~nm}$ when the FFP-TF is fixed at a certain wavelength. The instantaneous linewidth is $0.18 \mathrm{~nm}$ and the signal-to-noise ratio (SNR) of the laser is larger than $50 \mathrm{~dB}$. This high-quality lasing spectrum is due to the presence of the high-feedback-efficiency ring cavity around $1310 \mathrm{~nm}$. Figure 4 (b) shows the optical output spectrum (peak-hold mode) of the wavelength-swept cascaded Raman fiber laser when a triangularly modulated

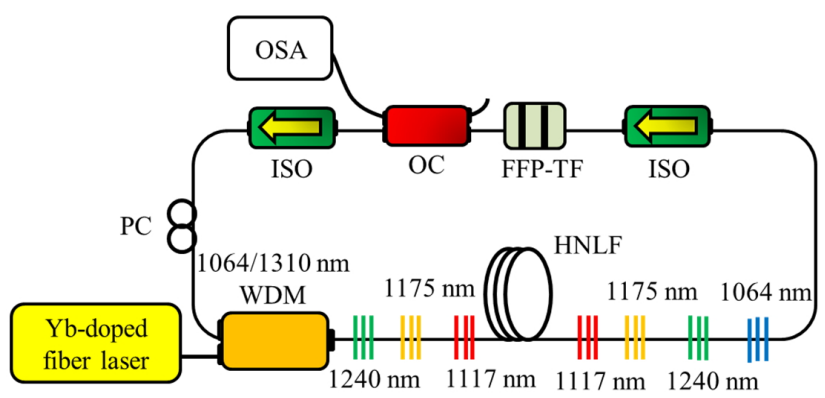

FIG. 3. Setup of the wavelength-swept cascaded Raman fiber laser based on cascaded Raman amplifier. (FFP-TF: Fiber Fabry-Perot Tunable Filter, OC: Output Coupler) driving signal of $100 \mathrm{~Hz}$ is applied to the FFP-TF. In this study, the measured sweeping range was $27 \mathrm{~nm}$ with the central wavelength of $1310 \mathrm{~nm}$, and the extinction ratio was approximately $43 \mathrm{~dB}$. The measured optical output power was $2.1 \mathrm{~mW}$ for a pumping power of $2.3 \mathrm{~W}$. Figure 4 (c) shows the temporal transient intensity profiles of the wavelengthswept laser output with a sweeping rate of $100 \mathrm{~Hz}$, which profiles were measured by an oscilloscope and a photodetector. This optical output performance of the wavelength-swept cascaded Raman fiber laser makes the laser suitable as the light source of the SS-OCT for operation around the 1310 $\mathrm{nm}$ region. The limitation of current sweeping speed and output power is mainly caused from the long length of fiber cavity for the Raman gain generation. Since the

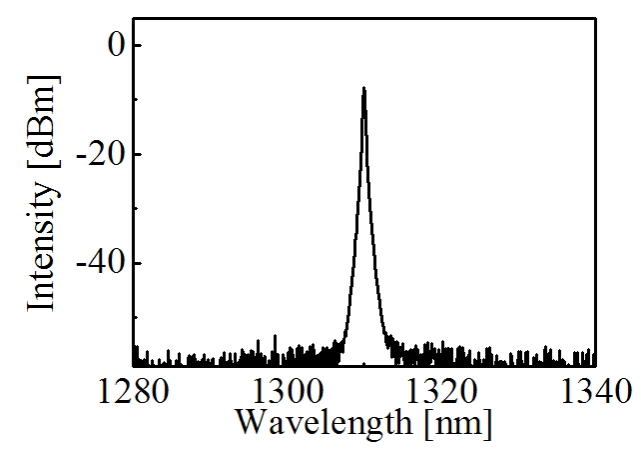

(a)

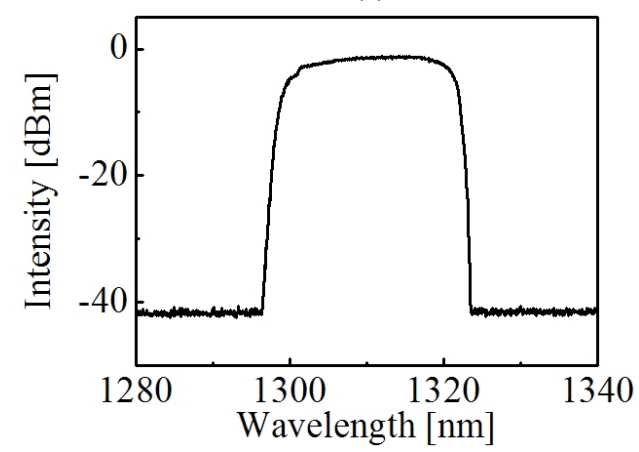

(b)

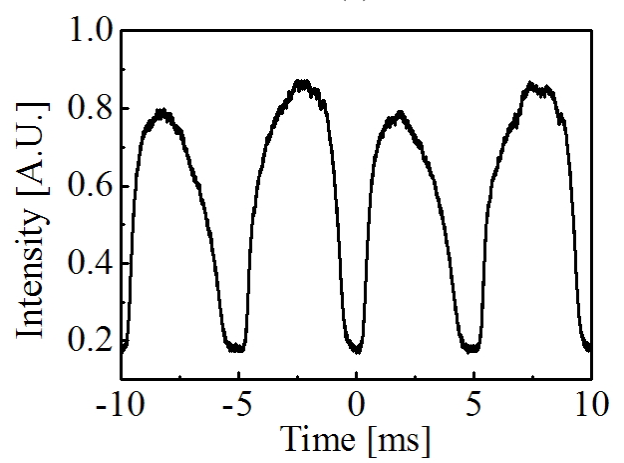

(c)

FIG. 4. (a) Output spectrum of stable laser without tuning of FFP-TF, (b) The peak-hold mode optical spectrum of the wavelength-swept laser with tuning of FFP-TF. (c) Temporal transient intensity profile of wavelength-swept output. 
roundtrip number is reduced for the longer length of HNLF and higher speed of FFP-TF, the output power will be decreased under the conventional wavelength-sweeping condition. The further investigations will be required to increase the sweeping speed by using the Fourier domain mode locked (FDML) method. When we use the optimized fiber length in cavity and corresponding speed of FFP-TF, the photon's round-trip time can be matched with repetition time of FFP-TF for the increase of the output power under the FDML condition [4].

\section{OCT IMAGING}

Figure 5 shows the experimental setup for SS-OCT imaging based on the proposed wavelength-swept cascaded Raman fiber laser. Two tunable directional couplers and two circulators were used to construct a Mach-Zehnder interferometer. The optimal optical power between the sample port and reflecting mirror port was controlled manually by means of the tunable directional coupler. A galvano mirror scanner was used for lateral scanning of the sample. The objective lens (LSM-03, Thorlabs) of 3x magnification and focal length of $54 \mathrm{~mm}$ was used for optimally gathering sample images. The converted reflecting intensities were recorded on a grayscale image as a function of the transverse and axial distances. Around 1024 depth points were acquired for the series of images, and each image was composed of 512 (axial) $\times 256$ (transverse) pixels.

Figure 6 (a) shows a depth-encoded signal obtained using the discrete Fourier transform (DFT) for a sample comprising three cover glasses (4science, thickness of $160 \mu \mathrm{m}$ ) [22]. The measured thickness was $159.9 \mu \mathrm{m}$ for the first cover glass. A two-dimensional (2D) OCT sample image was also obtained, as shown in Fig. 6 (b). All three cover glasses and the air gaps between the cover glasses can be clearly distinguished in the OCT image. The measured axial resolution was $28 \mu \mathrm{m}$, which exhibits reasonable agreement with the theoretical value. In addition, the measured transverse resolution was $39.7 \mu \mathrm{m}$. Figure 7 shows the cross-sectional OCT image

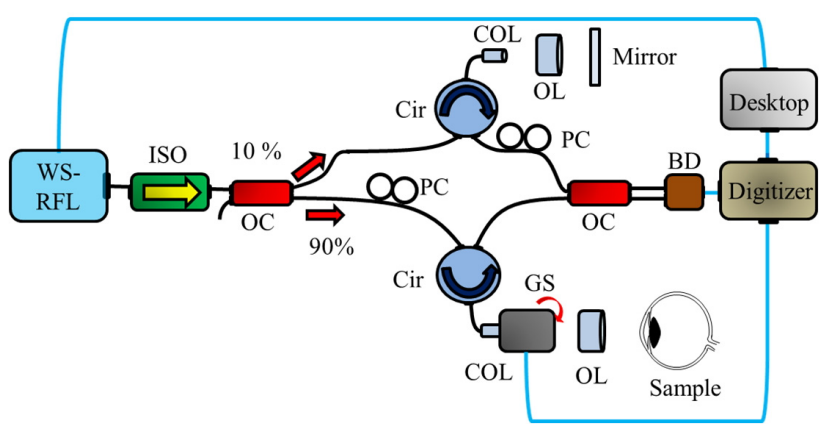

FIG. 5. Experimental setup for SS-OCT imaging using wavelength-swept cascaded Raman fiber laser. (WS-RFL: Wavelength-swept Raman Fiber Laser, OL: Objective Lens, COL: Collimator, GS: Galvano mirror Scanner, BD: Balanced Detector, Cir: Circulator, PC: Polarization Controller)

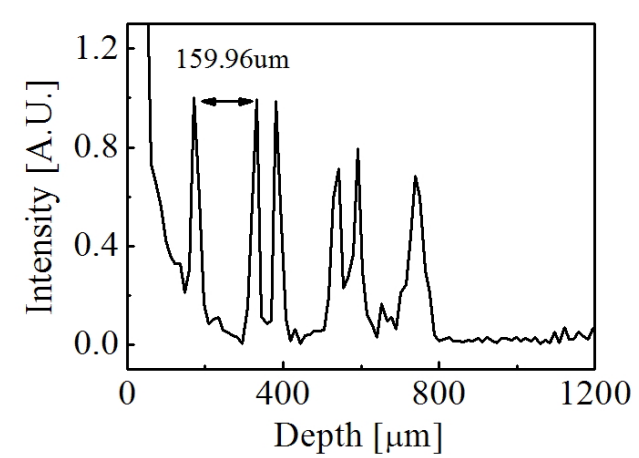

(a)

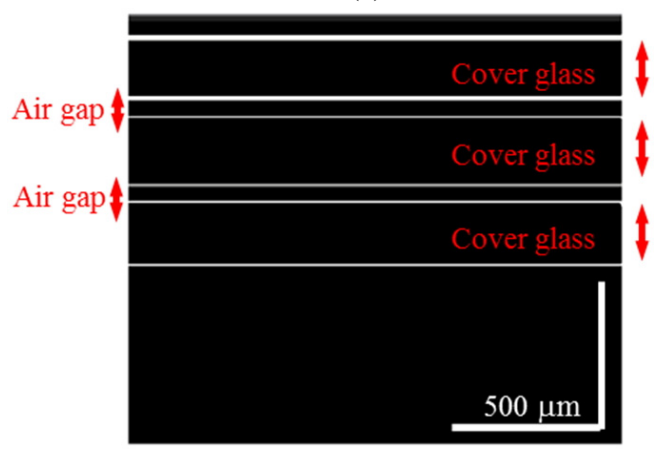

(b)

FIG. 6. (a) Depth encoded signal and (b) 2-dimensional OCT image of three cover glasses.

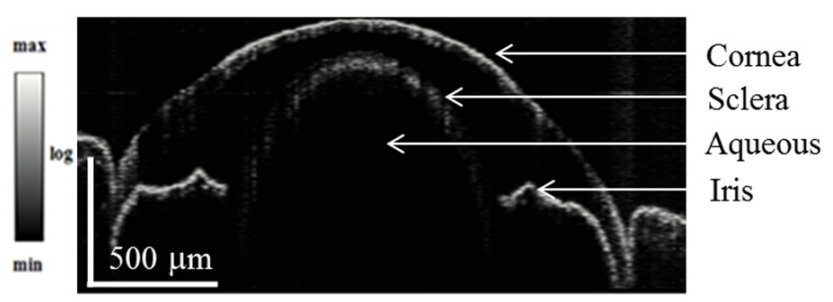

FIG. 7. Cross sectional OCT image of a fish eye sample.

of the eye sample of a small fish (Balantiocheilos melanopterus). A 2D OCT image was created from the Fourier transform of the corresponding $512 \times 256$ data set. The galvano scanner was driven at a frequency of $\sim 0.19 \mathrm{~Hz}$, and the acquisition time of the 2-D OCT image was $5.26 \mathrm{~s}$.

\section{CONCLUSION}

In this research, we proposed and experimentally demonstrated a wavelength-swept cascaded Raman fiber laser operating in the $1310 \mathrm{~nm}$ region with a $2 \mathrm{~km}$ long HNLF and low pump power of $2.3 \mathrm{~W}$. This was achieved using the optimal cavity structure of a 1064/1310 nm WDM coupler and coupled FBG mirrors reflecting wavelengths of 1064, 1117, 1175, and $1240 \mathrm{~nm}$. When the FFP-TF was operated with a $100 \mathrm{~Hz}$ triangularly modulated signal, the 
wavelength-swept cascaded Raman fiber laser output exhibited a sweep range over $27 \mathrm{~nm}$ and an output power of 2.1 $\mathrm{mW}$. When compared with the conventional wavelength-swept Raman laser source that is used for SS-OCT imaging around the 1550-nm region, we believe that our novel wavelengthswept cascaded Raman fiber laser operating around the $1310-\mathrm{nm}$ region can provided improved OCT imaging. The further investigation will be necessary to improve the output power and speed by the use of the FDML method and increase the sweeping range by the use of broader 3 $\mathrm{dB}$ bandwidth FBGs [4].

\section{ACKNOWLEDGMENT}

This research was supported by the Basic Science Research Program through the National Research Foundation of Korea (NRF) funded by the Ministry of Science, ICT and Future Planning (NRF-2013R1A1A1005761).

\section{REFERENCES}

1. S. H. Yun, G. J. Tearney, J. F. de Boer, N. Iftimia, and B. E. Bouma, "High-speed optical frequency-domain imaging," Opt. Express 11, 2953-2963 (2003).

2. R. Huber, M. Wojtkowski, and J. G. Fujimoto, "Fourier domain mode locking (FDML): A new laser operating regime and applications for optical coherence tomography," Opt. Express 14, 3225-3237 (2006)

3. M. Y. Jeon, J. Zhang, Q. Wang, and Z. Chen, "High-speed and wide bandwidth Fourier domain mode-locking wavelength swept laser with multiple SOAs," Opt. Express 16, 2547-2554 (2008).

4. E. J. Jeong, C. S. Kim, M. Y. Jeong, M. K. Kim, M. Y. Jeon, W. Jung, and Z. Chen, "Characterization of FBG sensor interrogation based on a FDML wavelength swept laser," Opt. Express 16, 16552-16560 (2008).

5. T. Wang, W. Wieser, G. Springeling, R. Beurskens, C. T. Lancee, T. Pfeiffer, A. F. W. van der Steen, R. Huber, and G. van Soest, "Intravascular optical coherence tomography imaging at 3200 frames per second," Opt. Lett. 38, 1715-1717 (2013).

6. W. Y. Oh, B. J. Vakoc, M. Shishkov, G. J. Tearney, and B. E. Bouma, "400 kHz repetition rate wavelength-swept laser and application to high-speed optical frequency domain imaging," Opt. Lett. 35, 2919-2921 (2010).

7. J. Xu, H. Ou, X. Wang, P. C. Chui, H. Y. Tam, and K. K. Y. Wong, "In vivo OCT imaging based on La-codoped Bismuth-based Erbium-doped fiber," IEEE Photon. Technol. Lett. 25, 1741-1743 (2013).

8. F. D. Nielsen, L. Thrane, J. Black, K. Hsu, A. Bjarklev, and P. E. Andersen, "Swept-wavelength sources for optical coherence tomography in the 1 um range," Proc. SPIE 5861,
632975 (2005).

9. K. H. Y. Cheng, B. A. Standish, V. X. D. Yang, K. Y. Cheung, X. Gu, E. Y. Lam, and K. K. Y. Wong, "Wavelengthswept spectral and pulse shaping utilizing hybrid Fourier domain modelocking by fiber optical parametric and erbiumdoped fiber amplifiers," Opt. Express 18, 1909-1915 (2010).

10. E. J. Jung, H. S. Lee, J. S. Park, M. Y. Jeong, and C. S. Kim, "Novel wavelength-swept Raman laser for arbitrary gain band OCT," Proc. SPIE 7168, 716823 (2009).

11. H. S. Lee, E. J. Jung, M. Y. Jung, and C. S. Kim, "Broadband wavelength-swept Raman laser for Fourier-domain mode locked swept-source OCT," J. Opt. Soc. Korea 13, 316-320 (2009).

12. T. Klein, W. Wieser, B. R. Biedermann, C. M. Eigenwillig, G. Palte, and R. Huber, "Raman-pumped Fourier-domain mode-locked laser: analysis of operation and application for optical coherence tomography," Opt. Lett. 33, 2815-2817 (2008).

13. J. Xu, H. Ou, X. Xu, V. X. D. Yang, P. C. Chui, and K. K. Y. Wong, "Wideband Raman-pumped wavelength-swept laser for optical coherence tomography application," Appl. Phys. Express 6, 062701 (2013).

14. N. Kurukitkoson, H. Sugahara, S. K. Turitsyn, O. N. Egorova, A. S. Kurkov, V. M. Paramonov, and E. M. Dianov, "Optimisation of two-stage Raman converter based on phosphosilicate core fibre: modelling and experiment," Electron. Lett. 37, 1281-1283 (2001).

15. M. Rini, I. Cristiani, V. Degiorgio, A. S. Kurkov, and V. M. Paramonov, "Experimental and numerical optimization of a fiber Raman laser," Opt. Commun. 203, 139-144 (2002).

16. S. A. Babin, D. V. Churkin, S. I. Kablukov, M. A. Rybakov, and A. A. Vlasov, "All-fiber widely tunable Raman fiber laser with controlled output spectrum," Opt. Express 15, 8438-8443 (2007).

17. F. Anquez, E. Courtade, A. SivÚry, P. Suret, and S. Randoux, "A high-power tunable Raman fiber ring laser for the investigation of singlet oxygen production from direct laser excitation around 1270 nm," Opt. Express 18, 22928-22936 (2010).

18. C. S. Kim and J. U. Kang, "Multiple wavelength switching of Raman fiber ring laser incorporating composite polarizationmaintaining fiber Lyot-Sagnac filter," Appl. Opt. 43, 3151-3157 (2004).

19. V. R. Supradeepa and J. W. Nicholson, "Power scaling of high-efficiency $1.5 \mu \mathrm{m}$ cascaded Raman fiber lasers," Opt. Lett. 38, 2538-2541 (2013).

20. K. Liu and E. Garmire, "Understanding the formation of the SRS stokes spectrum in fused silica fiber," IEEE J. Quantum Electron. 27, 1022-1030 (1991).

21. H. S. Seo, K. Oh, and U. C. Paek, "Gain optimization of germanosilicate fiber Raman amplifier and its applications in the compensation of Raman-induced crosstalk among wavelength division multiplexing channels," IEEE J. Quantum Electron. 37, 1110-1116 (2001).

22. J. S. Park, M. Y. Jeong, and C. S. Kim, "Post-tuning of sample position in common-path swept-source optical coherence tomography," J. Opt. Soc. Korea 15, 380-385 (2011). 